European Association for the

Development of Renewable Energies,

Environment and Power Quality (EA4EPQ)
International Conference on Renewable Energies and Power Quality (ICREPQ'11)

Las Palmas de Gran Canaria (Spain), 13th to 15th April, 2011

\title{
Smart meter based energy management system
}

\author{
Dr. Péter Kádár, member of IEEE \\ Dept. of Power Systems \\ Óbuda University \\ Bécsi u. 94. Budapest H-1034 HUNGARY \\ Phone: +36 209447 241; fax: +30 12500940 \\ peter.kadar@kvk.uni-obuda.hu
}

\begin{abstract}
.
The number of smart meters reached over 40 millions in Europe at the end of $2008^{1}$. Far beyond the simple energy metering, these devices enable companies to inform the customers, to control the load and to increase the energy consciousness.

The standardisation of the smart functionalities is in progress. The households for lack of knowledge and information do not care for the energy retrenchment.

New central software applications have been developed that can perform remote energy management functions through communication to the distributed smart meters (specific value calculation, trend monitoring, limit violation alarm, etc.). The paper gives an overview about the existing remote energy management systems and makes recommendations which functions would be useful in the Hungarian environment, too.
\end{abstract}

\section{Keywords}

Energy management system, smart meters, public energy consumers

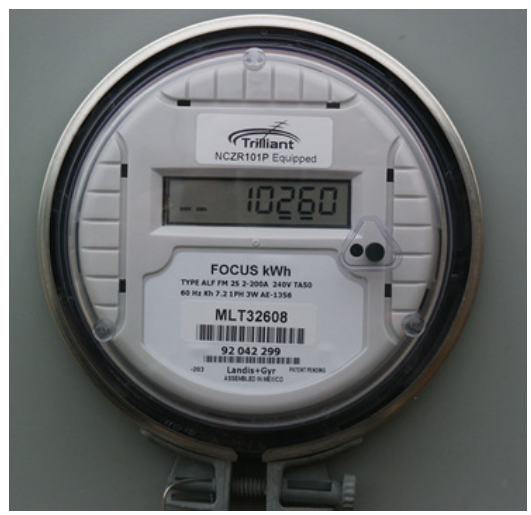

Figure 1. Trilliant smart meter ${ }^{2}$

\footnotetext{
${ }^{1}$ Berg Insight's Smart Metering in Western Europe report 2009

${ }^{2} \mathrm{http}$ ///www.miltonhydro.com/images/smart_meter.jpg
}

\section{Smart meters and their communication}

The smart energy meter redeems the traditional Ferrariswheel, electronic or digital meters. It has bidirectional communication capability for remote control and tariff based operation. The customer has up-to-date price, load and cost infos about gas-, water-, heat and electricity consumption. The smart meter is a basic end-user element of the smart grids, too.

The meters can have different communication tools:

a., The Zigbee network is one of the most widely used communication tools. ${ }^{3}$ The ZigBee Smart Energy supports the energy meterings, as the Home Display, remote programming of thermostats, lighting and load management.

$b$., The Home Area Network (HAN) operates in wireless and on-the-wire mode, too (e.g. Power Line Carrier, or IEEE 802.15.4 wireless protocol). The main device communicates with all the meters in the building and the gateway keeps contact with the Neighborhood Area Netwok (NAN).

c., The WiMAX (Worldwide Interoperability for Microwave Access) is a scalable bandwidth alternative of other communication channels based on 802.11n standard. ${ }^{4}$ Specifically serves the Intels and GE Energy's smart devices. ${ }^{5}$

On fig. 3. one can see the general, open smart communication system. Some Hungarian mobile telecommunication companies are going to develop their data transfer service in this structure. All the devices and communication methods can be integrated if they care for the necessary data content. Only the smart functionality is

\footnotetext{
${ }^{3} \mathrm{http} / /$ gigaom.com/cleantech/the-race-is-still-on-zigbee-vs-wifi-forsmart-energy-homes/

${ }^{4} \mathrm{http}: / / \mathrm{hu}$.wikipedia.org/wiki/WiMAX

${ }^{5} \mathrm{http} / / / \mathrm{www}$.onworld.com/html/newssmartmeter.htm
} 
defined and standardized, the protocols, tools, devices and options are free.

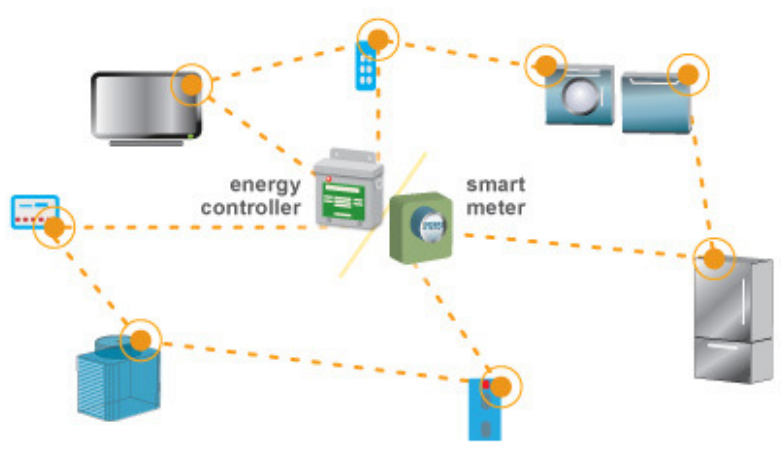

Fig. 2. Intelligent local communication ${ }^{6}$

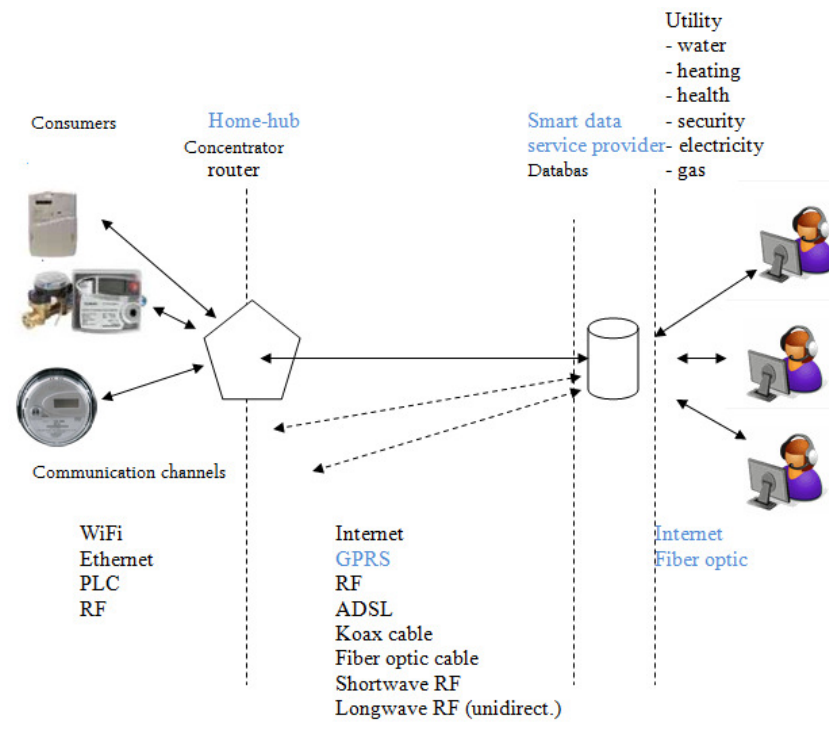

Fig. 3. Open Smart Communication ${ }^{7}$

\section{Energy management system}

The users concentrate on the economic gain, so the simplicity is really important.

The energy management system monitors and/or controls the load, the consumption devices. It informs the user about the tariff, about the energy consuming behaviour.

The ENERGYHUB is a full smart system that senses, controls and influences the network. EnergyHub handles different timing and programs for switching the lighting, heating, washing. The Web portal gives a lot of hints and advices on how to spare energy. It is also linked to community portals, such as Facebook.

${ }^{6}$ Intelligent tools http://www.miltonhydro.com/images/smart_meter.jpg

${ }^{7}$ Lecture book of informatics in energetics by Peter Kadar

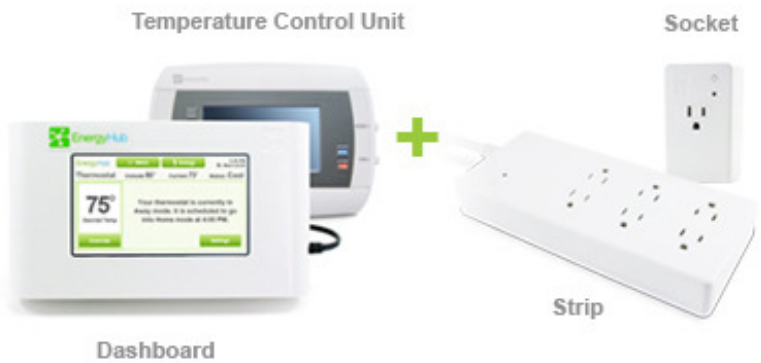

Fig 4. EnergyHub ${ }^{8}$

AGILEWAVES was developed by NASA engineers with the Building Optimization System (BOS) method. This web based system cooperates with different sensors, and monitors the HVAC devices (Heating, Ventilation, Air Conditioning) and furthermore the water and irrigations system, gas pipelines, precipitation, humidity, temperature, etc. It supports the renewable resources, such as solar collectors, PV, geothermal heating.

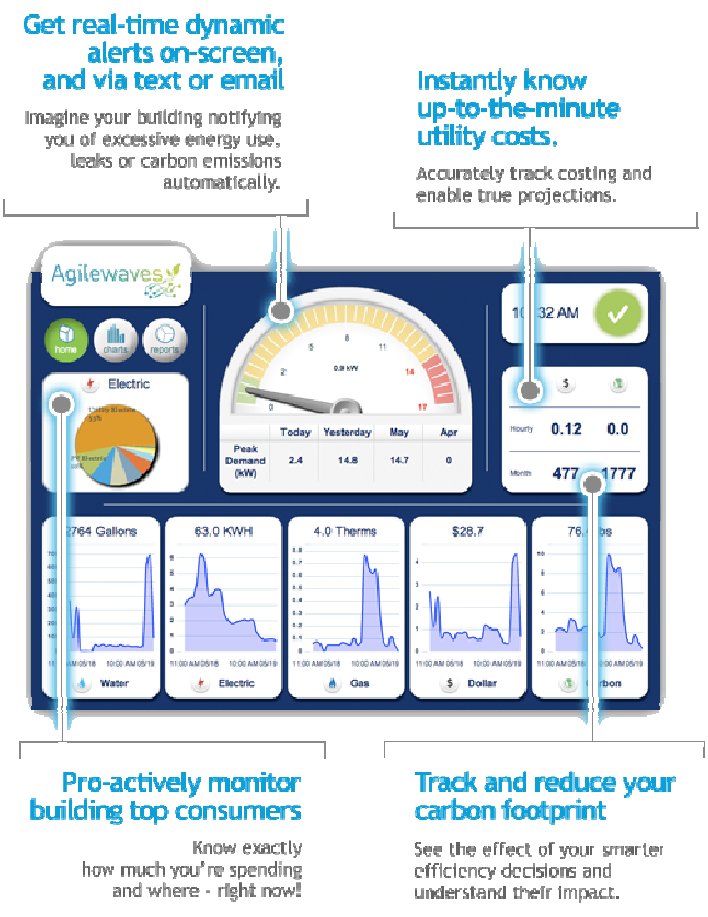

Fig. 5. Agilwaves ${ }^{9}$

GOOGLE POWERMETER cooperates with General Electric or Tendril devices. Open source web based platform ready to accept external development of functions with graphic presentation. This functionality is also available on handheld mobile tools.

\footnotetext{
${ }^{8}$ http://www.energyhub.com/wp-

content/uploads/2009/03/consumers_combo.jpg

${ }^{9} \mathrm{http}: / / \mathrm{www}$. agilewaves.com/agilewaves/wp-

content/uploads/2010/05/homepage-screenshot-v02.png
} 


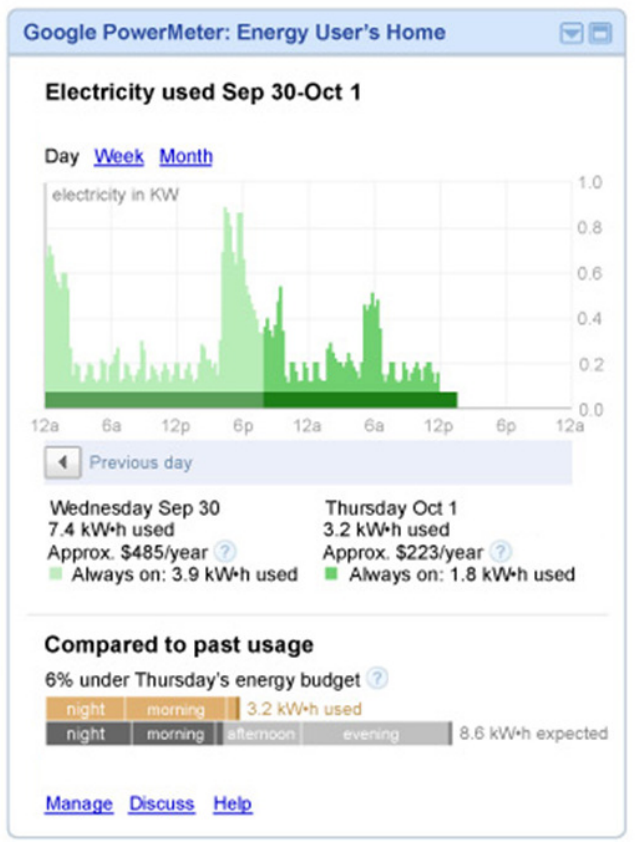

Fig. 6. Google PowerMeter ${ }^{10}$

\section{The practice of the energy consumption}

The municipial- and public institutions and the people consume without energy consciousness. Energy management belongs to non-technical departments, the proportion of the energy costs is really high. The possible centralization of bill paying makes the chance of energy saving.even worse.

Experiences at a municipality:

- The greatest loss is realized at the remote heating

- Energy consciousness would be needed

- The continuous monitoring would be needed

Characteristics of the public institutions:

- a lot of different loads (schools, hospital, museum, library, archive, social home for old people

- a lot of sites (a museum has 18 meters)

- different tariffs [base load (normal and ripple controlled), public, power dependent, etc.]

- low voltage level

- low consumption (cottage, garage, etc.)

- unpredictable and uncontrollable

- sites out of operation

- particular meters, tenants

- lack of specialist

- low load factor

- absence of utility contracts

- liability for public procurement procedure

\footnotetext{
${ }^{10} \mathrm{http}: / /$ www.google.com/powermeter/about/images/powermeter-uiinfo.jpg
}

The following figure shows the open energy purchase and consumption procedure that does not contain any feedback.
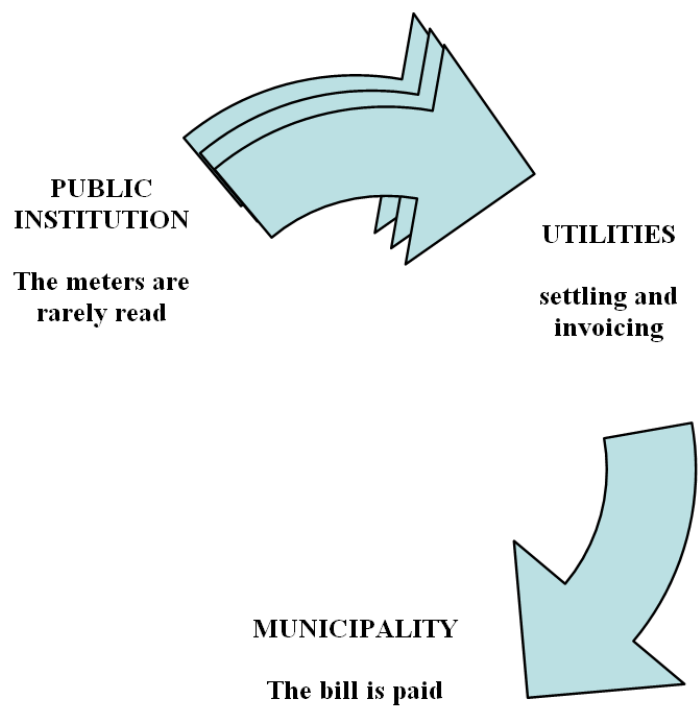

Fig 7. The present energy management process

\section{Proposed functionality}

The new basic concept of the remote energy management systems is that the public users without specific energy knowledge can handle and monitor the energy consumption of a distributed institution with more sites. It works on the basis of the data collected by smart meters. The information helps the owner to reduce the amount of the used energy, thus to reduce the energy costs.
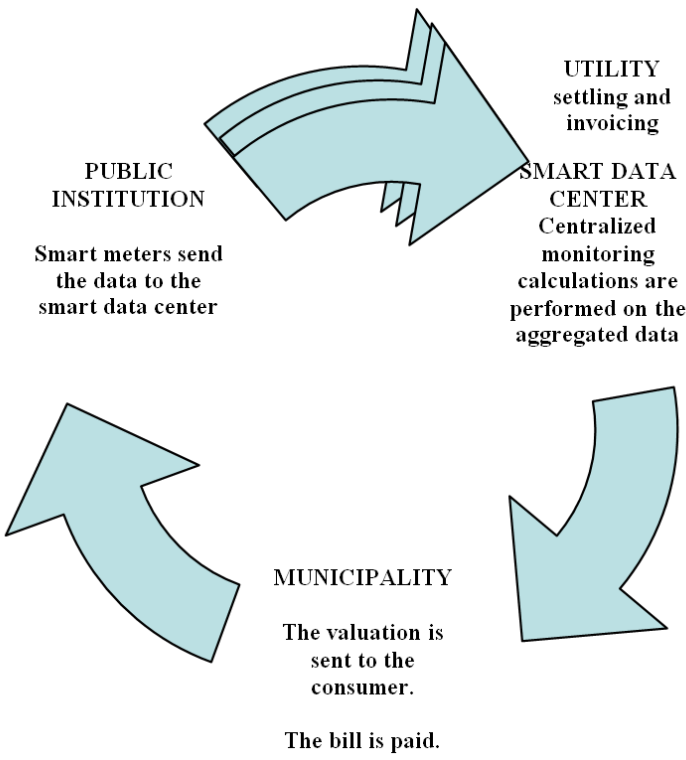

Fig 8 . The proposed energy management process

The following functionality is recommended for all types of energy, as gas, water, heat and electricity: 
- daily, weekly, monthly amount check and valuation of the consumption (normal, low, extraordinary, etc.)

- daily, weekly, monthly specific values

- limit violation alarm

- $\quad$ schedule and trend monitoring

- recommendation for better scheduling

- cost forecast

- $\quad$ saving potential calculation

- $\quad$ seasonal effects
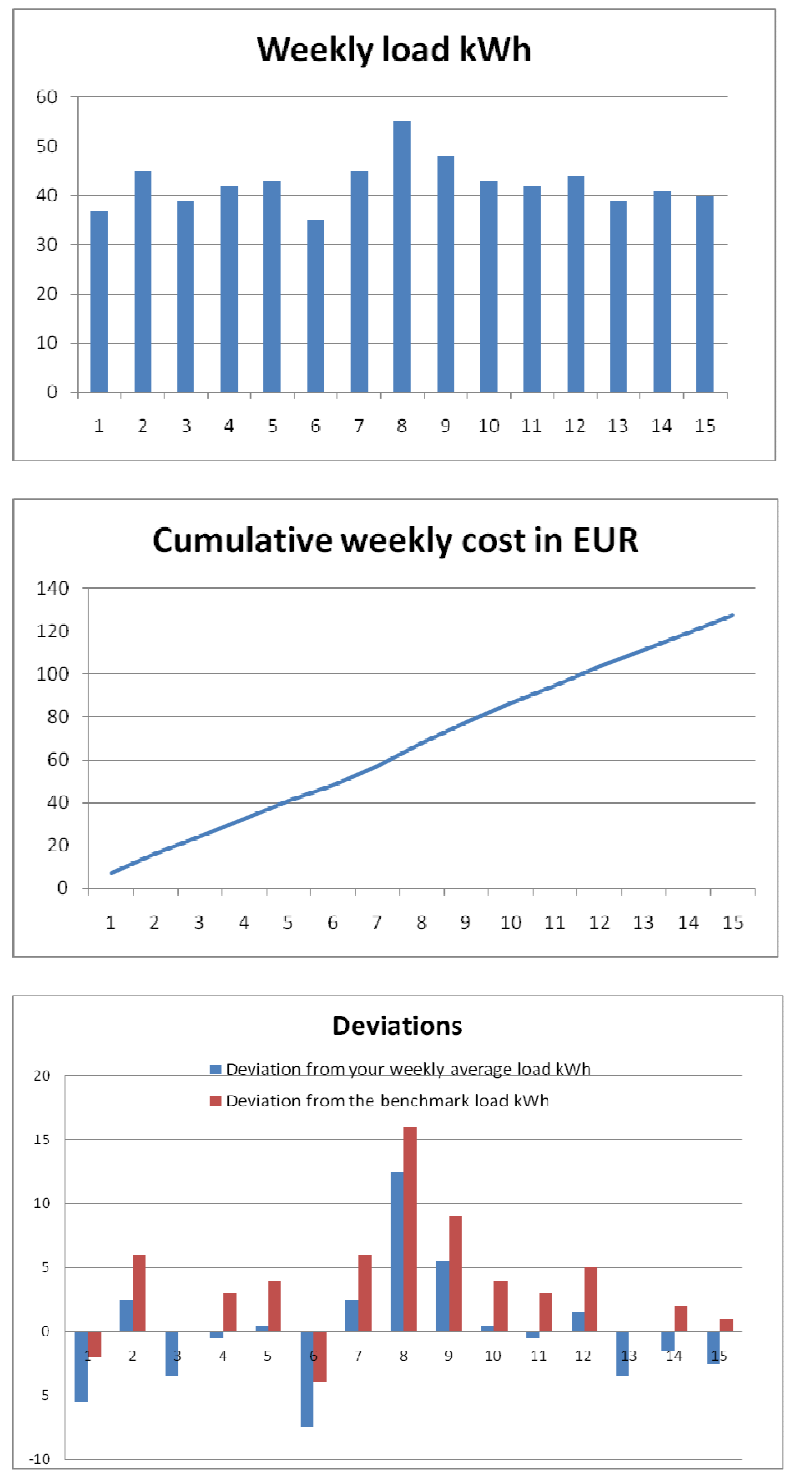

Fig. 9. The proposed energy management process

The energy management program can have personalized evaluation and recommendation about the customer's recent energy consumption habit, e.g.:

- Your weekly electricity cost is 28,45 EUR. It is 113,8 EUR per month or 1365,6 EUR per year. Up to this day in this year you consumed 475,3 EUR electricity. Do not be surprised to receive the bill!
- Over this weekend your consumption has been higher than a week before. Have you been at home?

- Your weekly load was $30 \%$ higher than the benchmark level.

- The peak temperature tomorrow will be $39{ }^{\circ} \mathrm{C}$. Please do not use your airconditioner at full power. Please make an air change in the early morning time. You can precool your flat.

- Use your washing machine only after $10 \mathrm{pm}$. You can save 0,32 EUR. It makes 15,36 EUR per year!

- Your night consumption was flat, but twice as high as one day ago. Did you turn off all the unnecessary lights during the night?

- The load neared $10 \%$ the contracted current values. In case of overstepping the fuse break the supply. Higher contracted values are recommended.

Fig. 9. shows possible individual screenshots of the Energy Management system. It provides a simple orientating overview about the most informative parameters. The customer information visualisation can be realized through internet and Personal Computer but the dedicated home display through smart meters' communication is more promising.

\section{Conclusions}

Some resistance can be detected against the spread of the smart meters in Hungary. In the paper a high level application possibility has been proposed, which makes these meters useful not only for the utility, but for the consumers and through the encouraging the energy saving for the national economy, as well.

\section{References}

[1] The Race Is (Still) On: ZigBee vs. WiFi for Smart Energy Homes http://gigaom.com/cleantech/the-raceis-still-on-zigbee-vs-wifi-for-smart-energy-homes/

[2] Zigbee Markets and Solutions Overview http://www.zigbee.org/Markets/Overview.aspx

[3] Trilliantic Wite Papers http://www.trilliantinc.com/library-files/whitepapers/HAN_white-paper.pdf

[4] Wikipédia Wimax http://hu.wikipedia.org/wiki/WiMAX

[5] On World: 100 Million New Smart Meters within the Next Five Years

http://www.onworld.com/html/newssmartmeter.htm

[6] Norten Ontario Wires

http://www.northernontariowires.com/smart_meters.ht $\mathrm{m}$

[7] Echelon Smart Grid Infrastructure http://www.echelon.com/metering/default.htm

[8] P. Kádár Péter - M. Polyák: Energy management and smart meters (in Hungarian); XXVI. Nemzetközi Kandó Konferencia 2010; Budapest, 2010. november 4.; ISBN 978-963-7158-04-9 